\title{
Optimization of a Single Phase Ultrasonic Linear Motor
}

\author{
Markus Flueckiger, José M. Fernandez, Manfred Giljum, and Yves Perriard, Senior Member IEEE \\ Integrated Actuators Laboratory - LAI \\ Ecole Polytechnique Fédérale de Lausanne - EPFL \\ CH - 1015 LAUSANNE - SWITZERLAND \\ Email : markus.flueckiger@epfl.ch
}

\begin{abstract}
Piezoelectric ultrasonic motors are superior to electromagnetic micromotors, because their efficiency remains theoretically constant during miniaturization. However, the still relatively recent technology has a considerable unexploited optimization potential. Numerical structural analysis by the means of the finite element method (FEM) is a common approach for dimensioning piezoelectric motors. Consequently, there is a need for efficient optimization procedures fitted to the FEM simulation. We developped a dedicated design methodology to first well understand the influence of the geometrical parameters on the movement of the motor. The parameters with the strongest influence on the objective function, the vibration amplitude of the resonator, were used in a following optimization stage. The operation of the optimized motor was proofed on a test bench. Interferometrical measurements validated quantitatively the FEM model along with the suggested design methodology.
\end{abstract}

\section{INTRODUCTION}

Piezoelectric actuators are nowadays established in industrial applications, where their well known advantages make them superior to electromagnetic micro motors. Nevertheless, certain reservations remain. Particularly control and drive electronics are often too complex and thus not economical for many potential application fields. We also observe that the majority of state of the art piezoelectric motors are driven by two or more phase shifted excitation signals [1]. Apart from developments on power electronics for piezoelectric actuators we therefore identify the need for motors with simplified working principles. Those should be controllable by basic electronics comparable to DC motor drives. Consequently we suggest a single phase linear piezoelectric motor, driven by a single low voltage sinusoidal signal.

This paper presents a design methodology for the finite element method (FEM) based development of new piezoelectric motors. It is then applied to the proposed linear motor with the goal of maximizing output speed. This is achieved by optimizing the resonator shape in order to increase the vibration amplitudes that cause the linear movement. First of all, a parametrization of the motor structure is carried out. Then, with the aim of reducing the number of simulations, but also to limit the variation ranges of the simulation parameters, a preoptimization stage is necessary. Thus, sensitivity analysis is carried out using design of experiments, which is a good way to obtain the influence of the input parameters on the objective function [2]. An optimization study, based on the results from preoptimization, is then realized using the Ansys FEM software. The resonator shapes obtained at each stage of this optimization process were fabricated and analyzed in order to validate the design methodology.

\section{Single Phase Motor Concept}

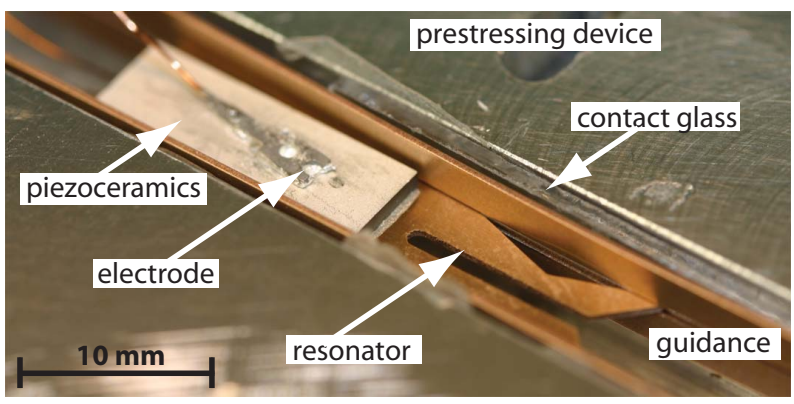

(a)

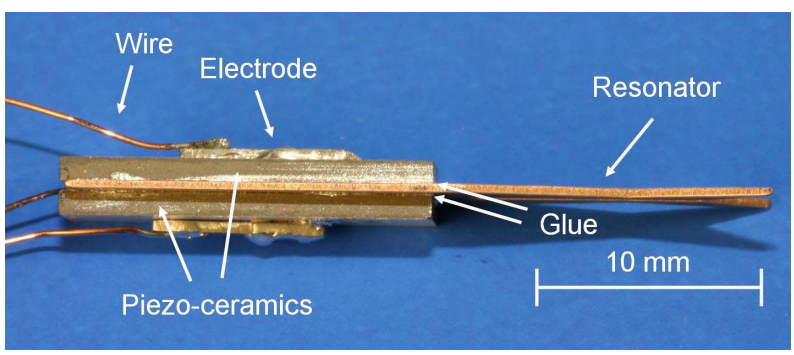

(b)

Figure 1. a) Functional model of the moleMotor; b) side view of the actuator

In Figure 1, a functional model of the moleMotor is shown. The actuator is placed within a flexible guidance which at the same time prestresses the resonator tips and guides the linear movement of the actuator. Unlike the motors using the direct working principle [3], the surface points at the resonator tips of the moleMotor that are in contact with the guidance do not perform an elliptical movement. Rather, the particular deformation of the resonator corresponds to a pushing or pulling movement respectively. The piezoelectric plates are placed such that the positive potentials are at the exterior surfaces and the negative potential comes in contact with the resonator. This configuration allows for stimulating two of the resonator's eigenmodes at the close by frequencies $84 \mathrm{kHz}$ and $69 \mathrm{kHz}$. The deformation of the eigenmode at $84 \mathrm{kHz}$ pushes 


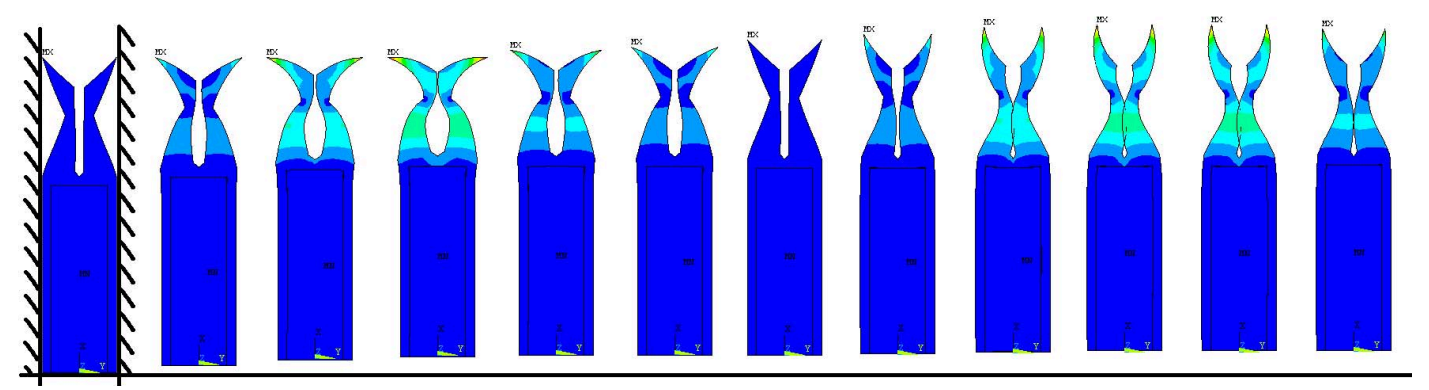

(a) Eigenmode at $84 \mathrm{kHz}$ corresponding to forward movement

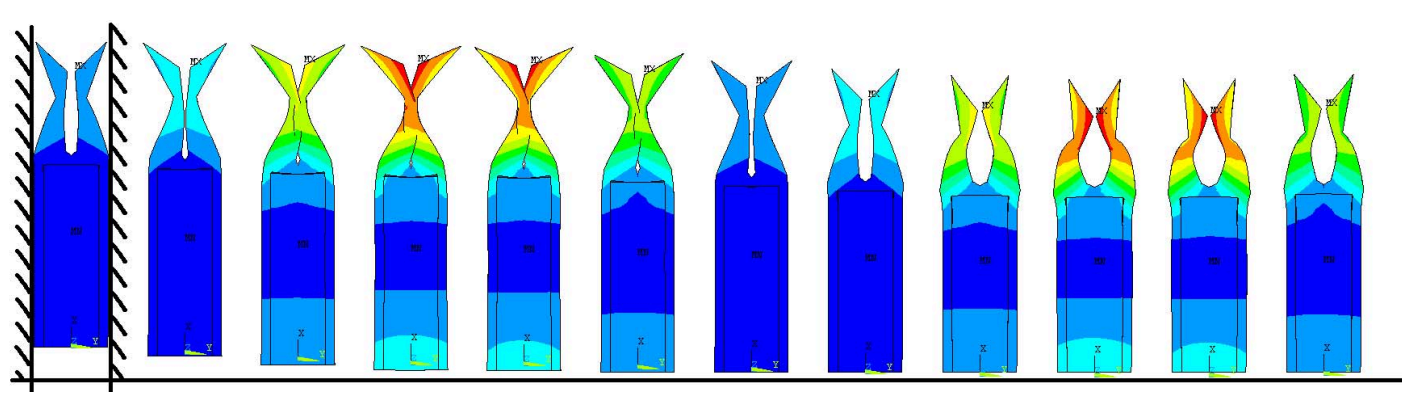

(b) Eigenmode at $69 \mathrm{kHz}$ corresponding to backward movement

Figure 2. Motion sequences of the moleMotor. The deformation amplitudes are strongly overdrawn.

the actuator forward. At the $69 \mathrm{kHz}$ eigenmode, the actuator is pulled backwards. Particularly, in the first part of a deformation cycle, the resonator tips bend towards the contact. Due to the frictional contact between the resonator tips and the guidance a force is created, causes the actuator to move in linear direction. When the resonator tips bend away from the guidance during the second part of the deformation cycle, contact is lost. The actuator continues to slide in the same direction because of the relatively high mass of the whole resonator compared to the mass of the tips. This working principle is illustrated in Figure 2. In the simulations that are displayed by these sequences, the contact was not simulated and the resonator was moving freely. To drive the motor, a single sinusoidal signal is applied to both piezoelectric elements simultaneously in order to excite the resonator's eigenmodes. To change direction, the frequency must be switched between $84 \mathrm{kHz}$ for forward motion and 69 $\mathrm{kHz}$ for backward motion.

\section{Numerical Modeling}

The FEM is widely used to model piezoelectric actuators, because it allows for computing complex structures with relatively little effort where accurate analytical models are difficult or even impossible to find.

The aim of numerical modeling is to calculate natural frequencies and modal shapes of the motor and to perform harmonic or transient response analysis. Basic equations for the motion of the motor can be written in matrix form (Equations 1 and 2) and are solved by applying the FEM [4].

$$
\begin{aligned}
{[M] \frac{\delta^{2}\{u\}}{\delta^{2} t}+[C] \frac{\delta\{u\}}{\delta t}+\left[K_{1}\right]\{u\}+\left[K_{2}\right]\{\Phi\} } & =\{F\} \\
{\left[K_{2}\right]^{T}\{u\}+\left[K_{3}\right]\{\Phi\} } & =[Q]
\end{aligned}
$$

where

$\{\mathbf{u}\},\{\boldsymbol{\Phi}\} \quad$ nodal displacement and potential vector;

$[\mathbf{M}],[\mathbf{C}]$ mass matrix and damping matrix;

$\left[\mathbf{K}_{\mathbf{1}, \mathbf{2}, \mathbf{3}}\right]$ stiffness, piezoelectric, dielectric matrices;

$\{\mathbf{F}\}$ nodal mechanical force vector;

$\{\mathbf{Q}\}$ electrical charge vector.

\section{A. FEM Model of the moleMotor}

FEM models approximate the real system. For the moleMotor we implemented electrical and mechanical properties according to Equations 1 and 2. The following assumptions were made:

- The piezoceramic plates are in direct contact to and immobile in reference to the resonator. The glue is taken into account by adjusting the damping factor of the resonator system.

- The electrodes glued on the piezoceramics and on the resonator have not been modeled. It was supposed that the outer surfaces of the piezoceramic plates are on positive and that the resonator is on negative electrical potential.

- As a first approach, the resonator tips are supposed to move freely.

Basically, it would seem judicious to directly maximize output force or speed of the motor by implementing a model of the contact phenomenon between stator and actuator tips. To reduce model complexity, however, we decided in a first approach to model only the actuator. Calculation time for FEM optimization can be significantly reduced in this way.

\section{B. Parametrization in Sight of Optimization}

The objective function of the optimization process is the deformation amplitude of the resonator with given piezoelec- 


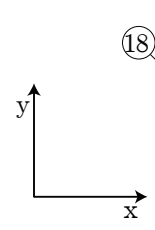

$\mathbf{a}_{1}$ : control

$\mathbf{a}_{\mathbf{4}}$ : res ky 7

$\mathbf{a}_{7}$ : res $\mathrm{kx} 10$

$\mathbf{a}_{\mathbf{1 0}}$ : res ky13

$\mathbf{a}_{13}$ : res $\mathrm{kx} 17$
(17)

$\mathbf{a}_{2}$ : voltage

$\mathbf{a}_{\mathbf{5}}$ : res $\mathrm{kx} 8$

$\mathbf{a}_{\mathbf{8}}$ : res ky10

$\mathbf{a}_{11}$ : res kx14

$\mathbf{a}_{\mathbf{1 4}}$ : res ky17

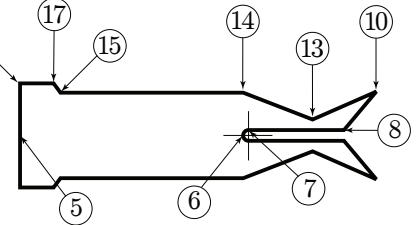

$\mathbf{a}_{3}$ : res $\mathrm{kx} 7$

$\mathbf{a}_{6}$ : res ky 8

$\mathbf{a}_{9}$ : res $\mathrm{kx} 13$

$\mathbf{a}_{\mathbf{1 2}}$ : res $\mathrm{kx} 15$

$\mathbf{a}_{15}$ : res $\mathrm{kx} 6$.
Figure 3. Definition of the resonator shape. The keypoints correspond to the main effects as indicated. "res $\mathrm{k}(\mathrm{x}, \mathrm{y}) i$ " designates the $\mathrm{x}$ and $\mathrm{y}$ positions respectively of the resonator keypoint $i$.

tric elements. Herewith we assume that the moleMotor's linear speed is directly proportional to the deformation amplitude of the resonator. This is apparent, as the excitation frequency remains constant during optimization and therefore a larger step size means higher speed. The free parameters in the design and optimization processes are the excitation voltage amplitude and the geometrical parameters defining the resonator shape. In Ansys the geometry was created by the connection of so called keypoints. During the simulations, the position of those keypoints and the excitation voltage were the only free parameters. They are implicitly bounded by the size of the piezoelectric elements. Figure 3 links the input parameters to the corresponding main effects of the sensitivity analysis.

\section{OPTIMIZATION}

The objectives of the preoptimization stage are two fold. On the one hand we aim to understand the influence of the different parameters on the objective function. Only those with an important influence are used for the optimization in order to reduce calculation time. On the other hand the fact to vary the parameter values for the sensitivity analysis leads already to a preoptimized motor design.

\section{A. Sensitivity Analysis}

The sensitivity analysis applied to the moleMotor optimization process consists in matching a taylor series approximation of the input function to the simulation results [5]:

$$
\begin{array}{r}
y(\bar{x})=a_{0}+\sum_{i=1}^{N} a_{i} x_{i}+\sum_{i \neq j}^{N} a_{i j} x_{i} x_{j}+\cdots \\
\sum_{i \neq j \neq k}^{N} a_{i j k} x_{i} x_{j} x_{k}+a_{i \ldots N} x_{i} \ldots x_{N}
\end{array}
$$

The coefficients represent the main effects $\left(a_{i}\right)$ and the interaction $\left(a_{i \ldots N}\right)$ of the system parameters (Figure 4$)$.

\section{B. FEM Optimization}

A FEM sweep optimization algorithm implemented in the Ansys software was used to maximize the vibration amplitude of the resonator tips and hence the motor speed. The information obtained with sensitivity analysis allowed for using
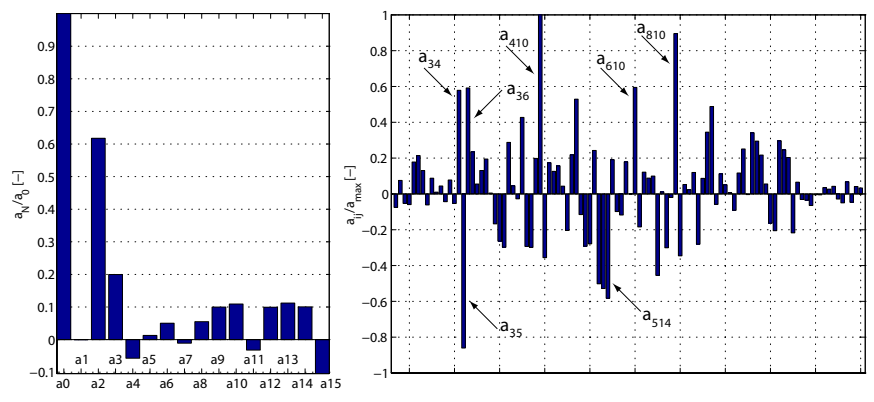

Figure 4. Sensitivity analysis: main effects and interaction

only a restricted set of model parameters without affecting the optimization results.

\section{Design Methodology Validation}

Functional models of the moleMotor were manufactured at the different stages of the design process.

Firstly, the comparison of simulation results to experimental measurements allowed for improving the numerical model. Because the motor consists of different materials and interfaces between them, the mechanical damping factor is difficult to determine analytically. Hence we started harmonic calulations on the FEM simulation model with an initial guess of $0.2 \%$. Then we used the experimental results from the functional models to adjust the damping factor accordingly. The final simulations were executed with a damping factor of $0.5 \%$.

Secondly, once the FEM simulation model was accurate, the results obtained from the functional models were compared to it and in this manner used to validate the presented design methodology.

\section{A. Interferometrical Vibration Amplitude Measurements}

Figure 5 compares the simulated and measured vibration amplitudes:

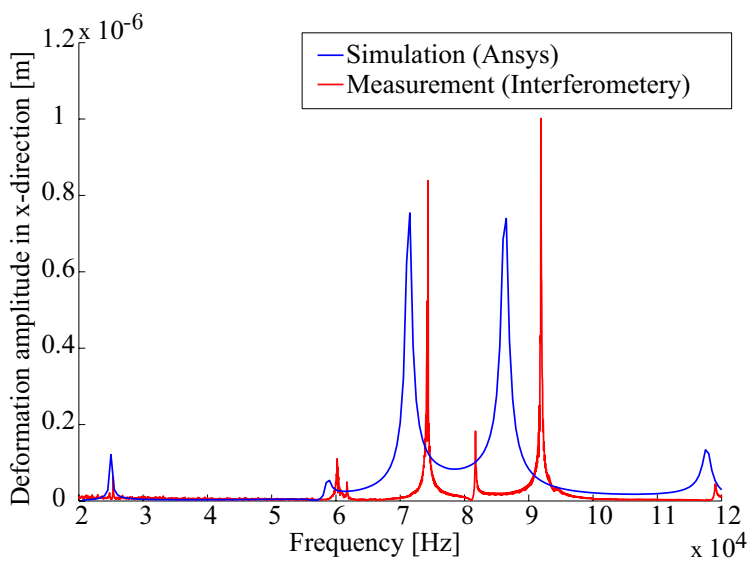

Figure 5. Optimized moleMotor: Comparison between experimental and simulated displacement amplitudes as a function of frequency exciting the piezoceramic with a $1.5 \mathrm{~V}$ signal

- Both graphs have qualitatively the same shapes and the same peaks. 


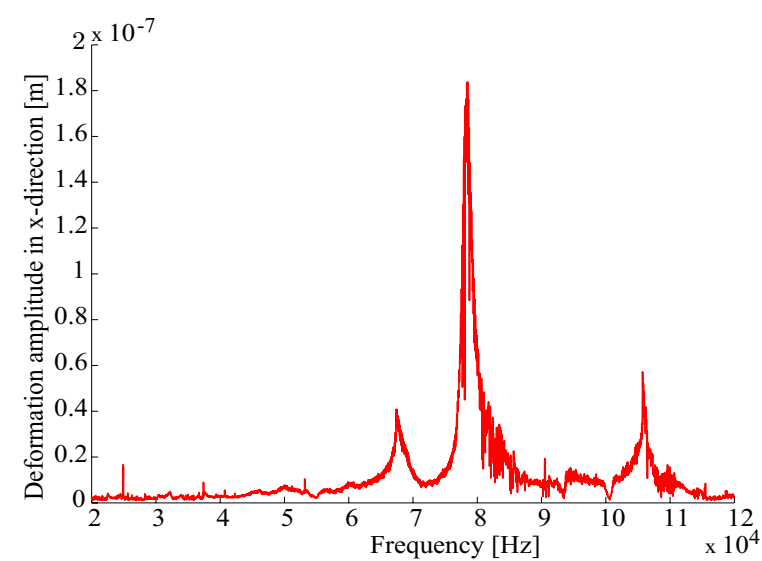

Figure 6. Optimized moleMotor: Measurement of the deformation amplitudes as a function of frequency for the preloaded functional model exciting the piezoceramic with a $21.5 \mathrm{~V}$ signal

- The simulation predicted resonance at about $5 \%$ lower frequencies.

- The vibration amplitudes are up to $20 \%$ larger in reality.

The observed disparity lies within the expected range and is caused by the following effects:

- Properties of piezoelectric materials are not constant among samples on the one hand and vary with external conditions such as ambient temperature on the other hand.

- Only a simplified actuator was modeled, which can explain some difference in frequency and the maxima of the deformation amplitude.

- The mechanical damping factor varies from model to model due to variable piezoelectric properties and manufacturing tolerances.

- In the interferometrical experiment the movement of the motor was not absolutely free. A small preload was still applied, in order to keep the motor in position.

These observations lead to the conclusion that the experiments did validate the FEM simulations as the results are qualitatively similar and quantitatively very close. The interferometrical measurements showed that the displacement amplitudes of the optimized structure are significantly bigger compared to the initial structure. Figure 6 shows that preload does not qualitatively change the vibration amplitudes. These results validate the optimization methodology.

\section{B. Optimization Results}

The methodical resonator shape optimization increased the deformation amplitude of the resonator tips to values up to six times larger compared to the results obtained from the initially guessed resonator shape. Figure 7 compares the vibration amplitudes at three different stages of the design process. The resonator shapes used for the first FEM simulation, and the ones obtained after preoptimization and after FEM optimization are shown.

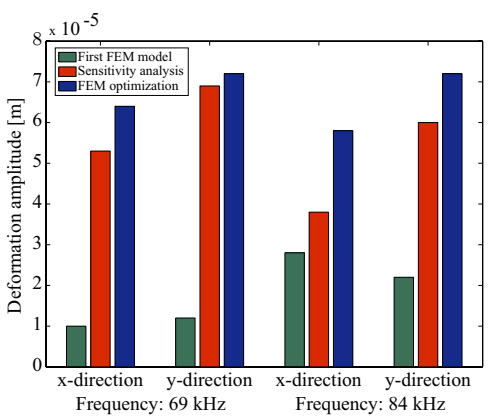

(a) Deformation amplitude of the actuators

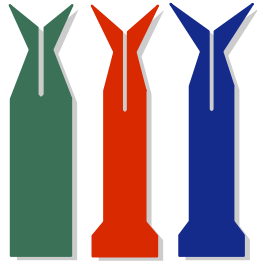

(b) Resonator shapes
Figure 7. Optimization results

\section{CONCLUSION}

The complementary design approach, using design of experiments in a preoptimization stage before applying FEM optimization algorithms to the motor model allows for vibration amplitude maximization. Calculation time of the optimization process is significantly reduced as only significant parameters are used for optimization and others excluded during preoptimization. Furthermore, the variation range of the significant parameters can be narrowed. Functional samples of motors corresponding to the initial, the preoptimized and the optimized structure have been built and tested on an experimental stage. The comparison of their characteristics to the predictions from the FEM model validated the design methodology. Nevertheless, an attractive extension would be to implement the contact phenomenon between actuator tips and stator in the FEM model in order to optimize speed and force output directly. The assumption that bigger deformation amplitudes lead to higher speed and force output does not take into account that output depends not only on the amplitude. The quality of the vibration is indeed very important. The trajectory of a surface point of the resonator tip when it is in contact with the guidance as well as stick and slip phenomena would be interesting to investigate.

\section{ACKNOWLEDGMENT}

The authors would like to thank Fabian Kägi at LSROEPFL and Claude Amendola at ATPR-EPFL for their advice on glueing and for resonator fabrication as well as Samuel Rosset and Renato Krpoun at LMTS-EPFL for their assistance with interferomety.

\section{REFERENCES}

[1] K. Spanner, "Survey of the various operating principles of ultrasonic piezomotors," in Actuator, 2006.

[2] G. Box, W. Hunter, and J. Hunter, Statistics for Experimenters, An introduction to design, data analysis and model building. Wiley, 1978.

[3] J. Wallaschek, "Piezoelectric ultrasonic motors," Journal of Intelligent Material Systems and Structures, vol. 6, no. 1, pp. 71-83, 1995.

[4] R. Lerch, "Simulation of piezoelectric devices by two- and threedimensional finite elements," IEEE Transactions on Ultrasonics, Ferroelectrics, and Frequency Control, vol. 37, no. 3, pp. 233-247, 1990.

[5] J. M. Fernandez and Y. Perriard, "Sensitivity analysis and optimization of a standing wave ultrasonic linear motor," IEEE Transactions on Ultrasonics, Ferroelectrics, and Frequency Control, vol. 53, no. 7, pp. 1352-1361, 2006. 